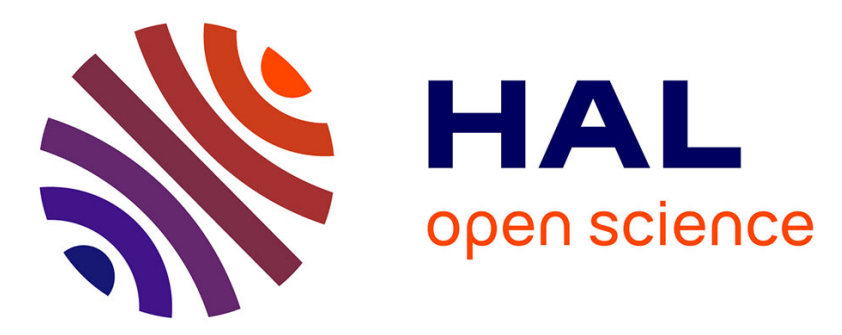

\title{
Rapid stabilization for a Korteweg-de Vries equation from the left Dirichlet boundary condition
}

Eduardo Cerpa, Jean-Michel Coron

\section{To cite this version:}

Eduardo Cerpa, Jean-Michel Coron. Rapid stabilization for a Korteweg-de Vries equation from the left Dirichlet boundary condition. IEEE Transactions on Automatic Control, 2013, 58 (7), pp.1688-1695. 10.1109/TAC.2013.2241479 . hal-00730190

\section{HAL Id: hal-00730190 https://hal.science/hal-00730190}

Submitted on 7 Sep 2012

HAL is a multi-disciplinary open access archive for the deposit and dissemination of scientific research documents, whether they are published or not. The documents may come from teaching and research institutions in France or abroad, or from public or private research centers.
L'archive ouverte pluridisciplinaire HAL, est destinée au dépôt et à la diffusion de documents scientifiques de niveau recherche, publiés ou non, émanant des établissements d'enseignement et de recherche français ou étrangers, des laboratoires publics ou privés. 


\title{
Rapid stabilization for a Korteweg-de Vries equation from the left Dirichlet boundary condition
}

\author{
Eduardo Cerpa and Jean-Michel Coron
}

\begin{abstract}
This paper deals with the stabilization problem for the Korteweg-de Vries equation posed on a bounded interval. The control acts on the left Dirichlet boundary condition. At the right end-point, Dirichlet and Neumann homogeneous boundary conditions are considered. The proposed feedback law forces the exponential decay of the system under a smallness condition on the initial data. Moreover, the decay rate can be tuned to be as large as desired. The feedback control law is designed by using the backstepping method.
\end{abstract}

Key words. Korteweg-de Vries equation, stabilization by feedback, backstepping AMS subject classifications. 35Q53, 93D15

\section{INTRODUCTION}

The Korteweg-de Vries (KdV) equation

$$
u_{t}(t, x)+u_{x}(t, x)+u_{x x x}(t, x)+u(t, x) u_{x}(t, x)=0,
$$

E. Cerpa is with the Departamento de Matemática, Universidad Técnica Federico Santa María, Avda. España 1680, Valparaíso, Chile. E-mail: eduardo.cerpa@usm.cl. This author was partially supported by the ERC advanced grant FP7-266907 CPDENL of the 7th Research Framework Programme (FP7), by Fondecyt grant \#11090161, by CMM Basal grant and by MathAmsud project CIP-PDE.

J.-M. Coron is with the Institut universitaire de France and Université Pierre et Marie Curie-Paris 6, UMR 7598, Laboratoire J.-L. Lions, Paris, F- 75005 France. E-mail: coron@ann.jussieu.fr. For this work, this author was supported by the ERC advanced grant FP7-266907 CPDENL of the 7th Research Framework Programme (FP7). 
posed on a bounded domain $[0, L]$ can be seen as a nonlinear control system where the inputs are the boundary data. From the nature of this equation, one boundary condition at the left end-point and two boundary conditions at the right end-point have to be imposed. The most studied case considers boundary conditions on

$$
u(t, 0), \quad u(t, L), \quad \text { and } \quad u_{x}(t, L) .
$$

Surprisingly, the control properties of this system are very different depending on where the controls are located. If we act on the left Dirichlet boundary condition and homogeneous data is considered at the right, then the system behaves like a heat equation and only null-controllability can be proven [22], [7]. On the other hand, if we act on the two right data and homogeneous boundary condition is considered at the left, then the system behaves like a wave equation with an infinite speed of propagation, in the sense that exact controllability holds for any time of control [21]. Another fascinating phenomena occurs when we put only one control input at the right end-point and keep homogeneous the other two boundary conditions: there exist some spatial domains (intervals of some given lengths) for which the corresponding linearized $\mathrm{KdV}$ equation is not any more controllable [21], [8]. In despite of that, in these critical cases the nonlinearity gives the exact controllability of the nonlinear $\mathrm{KdV}$ equation [5], [1], [2]. However, for some critical intervals the applied method requires a minimal time of control, which is not known to be really necessary.

Due to the existence of these critical intervals for the linear system, we do not know if the energy of the $\mathrm{KdV}$ equation is decreasing when the three boundary conditions considered are homogeneous (free control case). For instance, if $L=2 \pi$, the time-independent function given by $(1-\cos (x))$ satisfies

$$
u_{t}(t, x)+u_{x}(t, x)+u_{x x x}(t, x)=0, \quad u(t, 0)=u(t, 2 \pi)=u_{x}(t, 2 \pi)=0 .
$$

Therefore we find out a stationary solution of the linear $\mathrm{KdV}$ equation with homogeneous boundary conditions and consequently a solution with non-decreasing energy. This implies that 
for the free control case with $L=2 \pi$, the linearized system associated to the $\mathrm{KdV}$ equation (1) is not exponentially stable.

With a feedback control law acting at the left hand side such phenomenon does not appear and the method we propose in this case allows to address the problem of rapid exponential stabilization: given a desired decay rate, we find a feedback law exponentially stabilizing the system at that rate.

Based on the hyperbolic nature of the $\mathrm{KdV}$ equation controlled from the right, a method introduced in [10], [30] is used in [3] to get the rapid stabilization of the linear $\mathrm{KdV}$ equation from the Neumann boundary condition on the right. This gramian-based approach comes from the finite dimensional theory [18], [9] and was first introduced for PDE in the context of internal control [25]. This method requires the controllability of the linear system and therefore a non critical interval has to be considered.

In this paper, we applied the backstepping method to design the feedback control law. The backstepping method is very known as a ODE control method (see [11] and [4, Section 12.5]). The first extensions to PDE have appeared in [6] and [17]. Later on, Krstic and his collaborators introduced a modification of the method by means of an integral transformation of the PDE. This invertible transformation map the original PDE into an asymptotically stable one. In this context, the first continuous backstepping designs were proposed for the heat equation [16], [27]. The applications to wave equation appeared later in [12], [29], [26]. An excellent starting point to get inside this method is the book [13] by Krstic and Smyshlyaev.

This paper is organized as follows. In Section II] we formulate the problem and state the main result. Section [II] contains the backstepping design of the feedback control law. Section IV is devoted to prove the exponential decay of the $L^{2}$-norm of the solutions for the linearized system around the origin. In Section $\mathrm{V}$, we prove that this result still holds for the nonlinear $\mathrm{KdV}$ control system when the initial condition is small enough. Some extensions to the non-constant coefficient case and different boundary conditions are considered in Section VI. Finally, some final remarks are given in Section VII

Remark 1: The stabilization of the KdV equation by using an internal feedback control law 
was addressed in [20], [19] (see also [23], [15] for $\mathrm{KdV}$ equations with other nonlinearities). They have proved the following semi-global stabilization. Let $L>0, R>0$ and $a=a(x)$ a damping term satisfying $a(x) \geq a_{0}>0$, forall $x \in \omega$ where $\omega$ is nonempty open subset of $(0, L)$. Then, there exist $C=C(R)>0$ and $\mu=\mu(R)>0$ such that

$$
\|u(t, x)\|_{L^{2}(0, L)} \leq C e^{-\mu t}\|u(0, \cdot)\|_{L^{2}(0, L)}, \quad \forall t \geq 0
$$

for any solution of

$$
\left\{\begin{array}{l}
u_{t}(t, x)+u_{x}(t, x)+u_{x x x}(t, x)+a(x) u(t, x)+u(t, x) u_{x}(t, x)=0 \\
u(t, 0)=u(t, L)=u_{x}(t, L)=0
\end{array}\right.
$$

with $\|u(0, \cdot)\|_{L^{2}(0, L)} \leq R$. This result is different from ours in the sense that the exponential decay rate can not be imposed as large as desired. A key role in their design is played by the damping term $a=a(x)$, which prevents the existence of critical domains and allows to work with a dissipative system for any $L$. Other internal feedback control laws (static or time-varying ones) for the $\mathrm{KdV}$ equation with periodic boundary conditions can be found in [24], [14].

\section{Problem Statement and Main Result}

Given $L>0$, we consider the following nonlinear control system on the interval $[0, L]$

$$
\left\{\begin{array}{l}
u_{t}(t, x)+u_{x}(t, x)+u_{x x x}(t, x)+u(t, x) u_{x}(t, x)=0 \\
u(t, 0)=\kappa(t), \quad u(t, L)=0, \quad u_{x}(t, L)=0
\end{array}\right.
$$

For any positive $\lambda$, we address the problem of building some feedback control law $\kappa(t)=$ $\kappa(u(t, \cdot))$ such that the origin is exponentially stable for the corresponding closed-loop system (6) and the exponential decay rate is $\lambda$.

By using the backstepping method, we are able to find such a control law. The design is based on the linearized system around the origin. The linear closed-loop system is exponentially stable and the same result is obtained for the nonlinear $\mathrm{KdV}$ equation by adding a smallness condition on the initial data.

Our main theorem is the following. 
Theorem 2: For any $\lambda>0$, there exist a feedback control law $\kappa=\kappa(u(t, \cdot)), r>0$ and $D>0$ such that

$$
\|u(t, \cdot)\|_{L^{2}(0, L)} \leq D e^{-\lambda t}\|u(0, \cdot)\|_{L^{2}(0, L)}, \quad \forall t \geq 0
$$

for any solution of (6) satisfiying $\|u(0, \cdot)\|_{L^{2}(0, L)} \leq r$.

The feedback law $\kappa$ is explicitly defined as follows

$$
\kappa(t)=\int_{0}^{L} k(0, y) u(t, y) d y
$$

where the function $k=k(x, y)$ is characterized in Section III as the solution of a given partial differential equation depending on $\lambda$.

Remark 3: As we shall see in Section VI, Theorem 2 also holds if system (6) is replaced by

$$
\left\{\begin{array}{l}
u_{t}(t, x)+u_{x}(t, x)+u_{x x x}(t, x)+u(t, x) u_{x}(t, x)=0 \\
u(t, 0)=\kappa(t), \quad u_{x}(t, L)=0, \quad u_{x x}(t, L)=0
\end{array}\right.
$$

or

$$
\left\{\begin{array}{l}
u_{t}(t, x)+\gamma(x) u_{x}(t, x)+u_{x x x}(t, x)+u(t, x) u_{x}(t, x)+\delta(x) u(t, x)=0, \\
u(t, 0)=\kappa(t), \quad u(t, L)=0, \quad u_{x}(t, L)=0
\end{array}\right.
$$

where $\gamma, \delta$ are given functions in $C^{1}([0, L])$ and $C([0, L])$, respectively.

\section{Control Design}

The backstepping method applied here is based on the linear part of the equation. In this way, we consider the control system linearized around the origin

$$
\left\{\begin{array}{l}
u_{t}(t, x)+u_{x}(t, x)+u_{x x x}(t, x)=0 \\
u(t, 0)=\kappa(t), \quad u(t, L)=0, \quad u_{x}(t, L)=0
\end{array}\right.
$$

Given a positive parameter $\lambda$, we look for a transformation $\Pi: L^{2}(0, L) \rightarrow L^{2}(0, L)$ defined 
by

$$
w(x)=\Pi(u(x)):=u(x)-\int_{x}^{L} k(x, y) u(y) d y
$$

such that the trajectory $u=u(t, x)$, solution of 11 with

$$
\kappa(t)=\int_{0}^{L} k(0, y) u(t, y) d y
$$

is map into the trajectory $w=w(t, x)$, solution of the linear system

$$
\left\{\begin{array}{l}
w_{t}(t, x)+w_{x}(t, x)+w_{x x x}(t, x)+\lambda w(t, x)=0 \\
w(t, 0)=0, \quad w(t, L)=0, \quad w_{x}(t, L)=0
\end{array}\right.
$$

For system (14), called the target system, we have for any $t \geq 0$

$$
\frac{d}{d t} \int_{0}^{L}|w(t, x)|^{2} d x=-\left|w_{x}(t, 0)\right|^{2}-2 \lambda \int_{0}^{L}|w(t, x)|^{2} d x \leq-2 \lambda \int_{0}^{L}|w(t, x)|^{2} d x
$$

and therefore we easily obtain for $w=w(t, x)$ the exponential decay at rate $\lambda$

$$
\|w(t, \cdot)\|_{L^{2}(0, L)} \leq e^{-\lambda t}\|w(0, \cdot)\|_{L^{2}(0, L)}, \quad \forall t \geq 0
$$

In Section IV, we prove that, thanks to the invertibility of the map $\Pi$, the exponential decay (16) also holds for system (11).

Naturally, we can wonder if this decay rate is sharp. Let us notice that the eigenvalues of system (14) are the eigenvalues of

$$
\left\{\begin{array}{l}
w_{t}(t, x)+w_{x}(t, x)+w_{x x x}(t, x)=0 \\
w(t, 0)=0, \quad w(t, L)=0, \quad w_{x}(t, L)=0
\end{array}\right.
$$

shifted to the left $\lambda$ units. Thus, we are lead to study the eigenvalues $\sigma$ of

$$
\left\{\begin{array}{l}
-\phi^{\prime}(x)-\phi^{\prime \prime \prime}(x)=\sigma \phi(x) \\
\phi(0)=0, \quad \phi(L)=0, \quad \phi^{\prime}(L)=0
\end{array}\right.
$$

Surprisingly, the location of the eigenvalues of (18) depends on the length of the interval. 
From [21], we know that there exist some eigenvalues located on the imaginary axis if and only if $L \in\left\{2 \pi \sqrt{\frac{k^{2}+k \ell+\ell^{2}}{3}} /(k, \ell) \in \mathbb{N}^{2}\right\}$, which is called the set of critical lengths for this problem. In Figure 1, the first five eigenvalues of system (17) are plot for different values of $L$. In case (a), $L=1$ (non-critical) and the first eigenvalue $\sigma_{1}$ is approximately -72 . The system behaves like a dissipative one. In (b), $L=2 \pi$ (critical) and we have $\sigma_{1}=0$. The system has one conservative component given by the eigenfunction $\phi(x)=1-\cos (x)$. In (c), $L=2 \pi \sqrt{7 / 3}$ and the first two eigenvalues are imaginary numbers $\sigma_{1}=0.2 i$ and $\sigma_{2}=-0.2 i$. This examples show the different behaviors system (14) can have and the important role played by the parameter $\lambda$ in our design. In conclusion, the decay in 16 is optimal for some values of $L$.

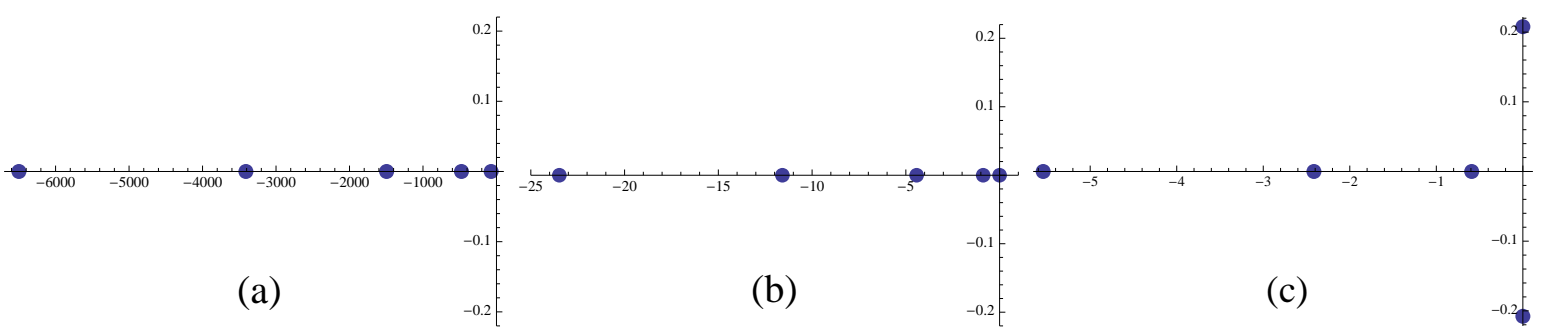

Figure 1. The first five eigenvalues of system 17 for (a) $L=1$, (b) $L=2 \pi$, and (c) $L=2 \pi \sqrt{7 / 3}$, respectively.

Let us focus in the key step, which is finding the kernel $k=k(x, y)$ such that $w(t, x)=$ $\Pi(u(t, x))$ satisfies $(14)$. For that, we perform the following computations

$$
\begin{aligned}
w_{t}(t, x)= & u_{t}(t, x)-\int_{x}^{L} u_{t}(t, y) k(x, y) d y \\
= & u_{t}(t, x)+\int_{x}^{L}\left(u_{y}(t, y)+u_{y y y}(t, y)\right) k(x, y) d y \\
= & u_{t}(t, x)-\int_{x}^{L} u(t, y)\left(k_{y}(x, y)+k_{y y y}(x, y)\right) d y \\
& -k(x, x)\left(u(t, x)+u_{x x}(t, x)\right) \\
& +k_{y}(x, x) u_{x}(t, x)-k_{y y}(x, x) u(t, x)+k(x, L) u(t, L) \\
& +k(x, L) u_{x x}(t, L)-k_{y}(x, L) u_{x}(t, L)+k_{y y}(x, L) u(t, L), \\
& \\
w_{x}(t, x)= & u_{x}(t, x)+k(x, x) u(t, x)-\int_{x}^{L} k_{x}(x, y) u(t, y) d y,
\end{aligned}
$$




$$
\begin{aligned}
w_{x x}(t, x)= & u_{x x}(t, x)+u(t, x) \frac{d}{d x} k(x, x)+k(x, x) u_{x}(t, x) \\
& +k_{x}(x, x) u(t, x)-\int_{x}^{L} k_{x x}(x, y) u(t, y) d y
\end{aligned}
$$

and

$$
\begin{aligned}
w_{x x x}(t, x)= & u_{x x x}(t, x)+u(t, x) \frac{d^{2}}{d x^{2}} k(x, x)+2 u_{x}(t, x) \frac{d}{d x} k(x, x) \\
& +k(x, x) u_{x x}(t, x)+u(t, x) \frac{d}{d x} k_{x}(x, x)+k_{x}(x, x) u_{x}(t, x) \\
& +k_{x x}(x, x) u(t, x)-\int_{x}^{L} k_{x x x}(x, y) u(t, y) d y
\end{aligned}
$$

Thus, given $\lambda \in \mathbb{R}$ and using (11), we have

$$
\begin{aligned}
& w_{t}(t, x)+w_{x}(t, x)+w_{x x x}(t, x)+\lambda w(t, x)= \\
& -\int_{x}^{L} u(t, y)\left(k_{x x x}(x, y)+k_{x}(x, y)+k_{y y y}(x, y)+k_{y}(x, y)+\lambda k(x, y)\right) d y \\
& \quad+k(x, L) u_{x x}(t, L)+u_{x}(t, x)\left(k_{y}(x, x)+k_{x}(x, x)+2 \frac{d}{d x} k(x, x)\right) \\
& \quad+u(t, x)\left(\lambda+k_{x x}(x, x)-k_{y y}(x, x)+\frac{d}{d x} k_{x}(x, x)+\frac{d^{2}}{d x^{2}} k(x, x)\right) .
\end{aligned}
$$

After the above computations and since $\frac{d}{d x} k(x, x)=k_{x}(x, y)+k_{y}(x, y)$, we obtain that one has (14) for every $u$ solution of (11) with (13) if the kernel $k=k(x, y)$ defined in the triangle $\mathcal{T}:=\{(x, y) / x \in[0, L], y \in[x, L]\}$ satisfies

$$
\left\{\begin{aligned}
k_{x x x}(x, y)+k_{y y y}(x, y)+k_{x}(x, y)+k_{y}(x, y) & =-\lambda k(x, y), & & \text { in } \mathcal{T}, \\
k(x, L) & =0, & & \text { in }[0, L], \\
k(x, x) & =0, & & \text { in }[0, L], \\
k_{x}(x, x) & =\frac{\lambda}{3}(L-x), & & \text { in }[0, L] .
\end{aligned}\right.
$$

Let us make the following change of variable

$$
t=y-x, \quad s=x+y,
$$


and define $G(s, t):=k(x, y)$. We have $k(x, y)=G(x+y, y-x)$ and therefore

$$
\begin{array}{r}
k_{x}=G_{s}-G_{t}, \quad k_{y}=G_{s}+G_{t}, \\
k_{x x}=G_{s s}-2 G_{s t}+G_{t t}, \quad k_{y y}=G_{s s}+2 G_{s t}+G_{t t}, \\
k_{x x x}=G_{s s s}-3 G_{s s t}+3 G_{s t t}-G_{t t t}, \\
k_{y y y}=G_{s s s}+3 G_{s s t}+3 G_{s t t}+G_{t t t} .
\end{array}
$$

Now, the function $G=G(s, t)$, defined in $\mathcal{T}_{0}:=\{(s, t) / t \in[0, L], s \in[t, 2 L-t]\}$, satisfies

$$
\left\{\begin{aligned}
6 G_{t t s}(s, t)+2 G_{s s s}(s, t)+2 G_{s}(s, t) & =-\lambda G(s, t), & & \text { in } \mathcal{T}_{0}, \\
G(s, 2 L-s) & =0, & & \text { in }[L, 2 L], \\
G(s, 0) & =0, & & \text { in }[0,2 L], \\
G_{t}(s, 0) & =\frac{\lambda}{6}(s-2 L), & & \text { in }[0,2 L] .
\end{aligned}\right.
$$

Let us transform this system into an integral one. We write the equation in variables $(\eta, \xi)$, integrate $\xi$ in $(0, \tau)$ and use that $6 G_{t s}(\eta, 0)=\lambda$. Next, we integrate $\tau$ in $(0, t)$ and use that $G_{s}(\eta, 0)=0$. Finally, we integrate $\eta$ in $(s, 2 L-t)$ and use that $G(2 L-t, t)=0$. Thus, we can write the following integral form for $G=G(s, t)$

$$
\begin{aligned}
G(s, t)=-\frac{\lambda t}{6}(2 L-t & -s) \\
& +\frac{1}{6} \int_{s}^{2 L-t} \int_{0}^{t} \int_{0}^{\tau}\left(2 G_{s s s}(\eta, \xi)+2 G_{s}(\eta, \xi)+\lambda G(\eta, \xi)\right) d \xi d \tau d \eta
\end{aligned}
$$

To prove that such a function $G=G(s, t)$ exists, we use the method of successive approximations. We take as an initial guess

$$
G^{1}(s, t)=-\frac{\lambda t}{6}(2 L-t-s)
$$

and define the recursive formula as follows,

$$
G^{n+1}(s, t)=\frac{1}{6} \int_{s}^{2 L-t} \int_{0}^{t} \int_{0}^{\tau}\left(2 G_{s s s}^{n}(\eta, \xi)+2 G_{s}^{n}(\eta, \xi)+\lambda G^{n}(\eta, \xi)\right) d \xi d \tau d \eta
$$


Performing some computations, we get for instance

$$
G^{2}(s, t)=\frac{1}{108}\left\{t^{3}\left(\lambda-\lambda^{2} L+\frac{\lambda^{2} t}{4}\right)(2 L-t-s)+\frac{t^{3} \lambda^{2}}{4}\left[(2 L-t)^{2}-s^{2}\right]\right\}
$$

and more generally the following formula

$$
G^{k}(s, t)=\sum_{i=1}^{k}\left(a_{k}^{i} t^{2 k-1}+b_{k}^{i} t^{2 k}\right)\left[(2 L-t)^{i}-s^{i}\right],
$$

where the coefficients satisfy $b_{k}^{k}=0$ and more importantly, there exist positive constants $M, B$ such that, for any $k \geq 1$ and any $(s, t) \in \mathcal{T}_{0}$

$$
\left|G^{k}(s, t)\right| \leq M \frac{B^{k}}{(2 k) !}\left(t^{2 k-1}+t^{2 k}\right)
$$

This implies that the series $\sum_{n=1}^{\infty} G^{n}(s, t)$ is uniformly convergent in $\mathcal{T}_{0}$. Therefore the series defines a continuous function $G: \mathcal{T}_{0} \rightarrow \mathbb{R}$

$$
G(s, t)=\sum_{n=1}^{\infty} G^{n}(s, t)
$$

and we get a solution of our integral equation. Indeed, we can write

$$
\begin{aligned}
& G=G^{1}+\sum_{n=1}^{\infty} G^{n+1} \\
&= G^{1}+\frac{1}{6} \sum_{n=1}^{\infty} \int_{s}^{2 L-t} \int_{0}^{t} \int_{0}^{\tau}\left(2 G_{s s s}^{n}(\eta, \xi)+2 G_{s}^{n}(\eta, \xi)+\lambda G^{n}(\eta, \xi)\right) d \xi d \tau d \eta \\
&=G^{1}+\frac{1}{6} \int_{s}^{2 L-t} \int_{0}^{t} \int_{0}^{\tau}\left(2 \sum_{n=1}^{\infty} G_{s s s}^{n}(\eta, \xi)+2 \sum_{n=1}^{\infty} G_{s}^{n}(\eta, \xi)+\lambda \sum_{n=1}^{\infty} G^{n}(\eta, \xi)\right) d \xi d \tau d \eta \\
&=G^{1}+\frac{1}{6} \int_{s}^{2 L-t} \int_{0}^{t} \int_{0}^{\tau}\left(2 G_{s s s}(\eta, \xi)+2 G_{s}(\eta, \xi)+\lambda G(\eta, \xi)\right) d \xi d \tau d \eta
\end{aligned}
$$

where we have used that the corresponding series $\sum_{n \geq 1} G_{s}^{n}$ and $\sum_{n \geq 1} G_{s s s}^{n}$ are also uniformly convergent.

Once we have found the function $G=G(s, t)$, we get the existence of the kernel $k=k(x, y)$. It is easy to see that the map $\Pi: L^{2}(0, L) \rightarrow L^{2}(0, L)$, defined by $(12)$, is continuous and 
consequently we have the existence of a positive constant $D_{\kappa}$ such that

$$
\|\Pi(f)\|_{L^{2}(0, L)} \leq D_{\kappa}\|f\|_{L^{2}(0, L)}, \quad \forall f \in L^{2}(0, L) .
$$

In Figure 2, we plot the gain kernel $k(0, y)$ (see (12)) as a function of $y \in[0, L]$ for different lengths (a) $L=1$ (non-critical), (b) $L=2 \pi$ (critical) and (c) $L=2 \pi \sqrt{7 / 3}$ (critical). The kernel functions are defined with $\lambda=1$. This illustrate the fact that case (a) is easier to stabilizes than case (b), which is easier to stabilizes than case (c). This is due to the location of the corresponding open-loop eigenvalues as shown in Figure 1.

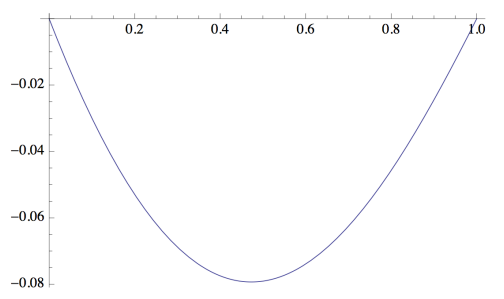

(a)

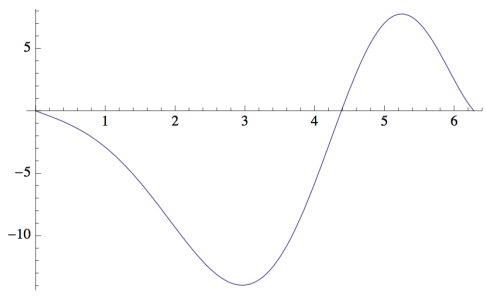

(b)

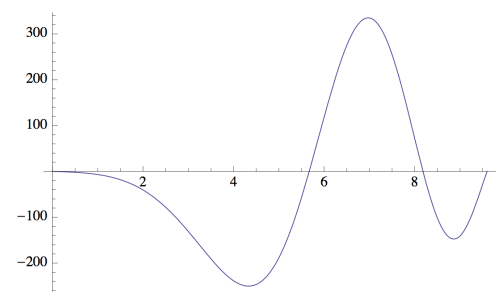

(c)

Figure 2. Gain kernel $k(0, y)$ corresponding to $\lambda=1$ for (a) $L=1$, (b) $L=2 \pi$, and (c) $L=2 \pi \sqrt{7 / 3}$, respectively.

\section{STABILITY OF THE LINEAR SYSTEM}

We know that the target system (14) is exponentially stable. In order to get the same conclusion for the linear system (11), the method we are applying uses the inverse transformation $\Pi^{-1}$. For that, we introduce a kernel function $\ell(x, y)$ which satisfies

$$
\left\{\begin{aligned}
\ell_{x x x}(x, y)+\ell_{y y y}(x, y)+\ell_{x}(x, y)+\ell_{y}(x, y) & =\lambda \ell(x, y), & & \text { in } \mathcal{T}, \\
\ell(x, L) & =0, & & \text { in }[0, L], \\
\ell(x, x) & =0, & & \text { in }[0, L], \\
\ell_{x}(x, x) & =\frac{\lambda}{3}(L-x), & & \text { in }[0, L],
\end{aligned}\right.
$$

The existence and uniqueness of such a kernel $\ell=\ell(x, y)$ are proven in the same way than for the kernel $k=k(x, y)$ in Section III. Once we have defined $\ell=\ell(x, y)$, it is easy to see that 
the transformation $\Pi^{-1}$ is characterized by

$$
u(x)=\Pi^{-1}(w(x)):=w(x)+\int_{x}^{L} \ell(x, y) w(y) d y .
$$

Let us see that $k(x, y)$ and $\ell(x, y)$ are related by the formula

$$
\ell(x, y)-k(x, y)=\int_{x}^{y} k(x, \eta) \ell(\eta, y) d \eta
$$

which in fact proves that $\Pi^{-1}$ maps a trajectory of $(14)$ into a trajectory of 11 with control $\kappa(t)$ defined by (13). Indeed, by plugging (41) into (12) and using Fubini's theorem we get, for any $w \in L^{2}(0, L)$, that for any $x \in[0, L]$

$$
\int_{x}^{L}(\ell(x, y)-k(x, y)) w(y) d y=\int_{x}^{L} \int_{x}^{y} k(x, \eta) \ell(\eta, y) w(y) d \eta d y
$$

which proves (42) for any $(x, y) \in \mathcal{T}$.

The map $\Pi^{-1}: L^{2}(0, L) \rightarrow L^{2}(0, L)$ is continuous and therefore we get the existence of a positive constant $D_{\ell}$ such that

$$
\left\|\Pi^{-1}(f)\right\|_{L^{2}(0, L)} \leq D_{\ell}\|f\|_{L^{2}(0, L)}, \quad \forall f \in L^{2}(0, L) .
$$

Let us prove that system (11)-(13) is exponentially stable. In fact, given $u_{0} \in L^{2}(0, L)$, we define

$$
w_{0}(x)=\Pi\left(u_{0}(x)\right):=u_{0}(x)-\int_{x}^{L} k(x, y) u_{0}(y) d y .
$$

The solution of (14) with initial condition $w(0, x)=w_{0}(x)$ satisfies $[15)$, i.e.

$$
\|w(t, \cdot)\|_{L^{2}(0, L)} \leq e^{-\lambda t}\left\|w_{0}(\cdot)\right\|_{L^{2}(0, L)}, \quad \forall t \geq 0 .
$$

Moreover, the solution of (11) is given by $u(t, x)=\Pi^{-1}(w(t, x))$. Thus, from (39), (44) and 
(46) we have for any $t \geq 0$

$$
\begin{aligned}
\|u(t, \cdot)\|_{L^{2}(0, L)} \leq D_{\ell}\|w(t, \cdot)\|_{L^{2}(0, L)} & \\
& \leq D_{\ell} e^{-\lambda t}\left\|w_{0}(\cdot)\right\|_{L^{2}(0, L)} \leq D_{\ell} D_{k} e^{-\lambda t}\left\|u_{0}(\cdot)\right\|_{L^{2}(0, L)}
\end{aligned}
$$

which proves the exponential decay at rate $\lambda$ for system (11) with feedback law (13).

\section{Stability of The NONLinear System}

Let $u=u(t, x)$ be a solution of the nonlinear equation (6) with the control $\kappa$ given by (13). Then, $w=\Pi(u(t, x))$ satisfies

$$
\begin{aligned}
& w_{t}(t, x)+w_{x}(t, x)+w_{x x x}(t, x)+\lambda w(t, x) \\
&=-\left(w(t, x)+\int_{x}^{L} \ell(x, y) w(t, y) d y\right)\left(w_{x}(t, x)+\int_{x}^{L} \ell_{x}(x, y) w(t, y) d y\right)
\end{aligned}
$$

with homogeneous boundary conditions

$$
w(t, 0)=0, \quad w(t, L)=0, \quad w_{x}(t, L)=0 .
$$

We multiply 48 by $w$ and integrate in $(0, L)$ to obtain

$$
\frac{d}{d t} \int_{0}^{L}|w(t, x)|^{2} d x=-\left|w_{x}(t, 0)\right|^{2}-2 \lambda \int_{0}^{L}|w(t, x)|^{2} d x-2 \int_{0}^{L} w(t, x) F(t, x) d x
$$

where the term $F=F(t, x)$ is given by

$$
\begin{aligned}
F(t, x)=w(t, x) \int_{x}^{L} \ell_{x}(x, y) w(t, y) & d y+w_{x}(t, x) \int_{x}^{L} \ell(x, y) w(t, y) d y \\
& +\left(\int_{x}^{L} \ell(x, y) w(t, y) d y\right)\left(\int_{x}^{L} \ell_{x}(x, y) w(t, y) d y\right) .
\end{aligned}
$$

We can prove that there exists a positive constant $C=C\left(\|\ell\|_{C^{1}(\mathcal{T})}\right)$ such that

$$
\left|2 \int_{0}^{L} w(t, x) F(t, x) d x\right| \leq C\left(\int_{0}^{L}|w(t, x)|^{2}\right)^{3 / 2}
$$


and therefore, if there exists $t_{0} \geq 0$ such that

$$
\left\|w\left(t_{0}, \cdot\right)\right\|_{L^{2}(0, L)} \leq \frac{\lambda}{C}
$$

then we obtain

$$
\frac{d}{d t} \int_{0}^{L}|w(t, x)|^{2} d x \leq-\lambda \int_{0}^{L}|w(t, x)|^{2} d x, \quad \forall t \geq t_{0}
$$

Thus, we get

$$
\|w(t, \cdot)\|_{L^{2}(0, L)} \leq e^{-\frac{\lambda}{2} t}\|w(0, \cdot)\|_{L^{2}(0, L)}, \quad \forall t \geq 0
$$

provided that

$$
\|w(0, \cdot)\|_{L^{2}(0, L)} \leq \frac{\lambda}{C}
$$

As we did for the linear system, by using the continuity of the transformations $\Pi$ and $\Pi^{-1}$ (see (39) and (44) and (55), we obtain the exponential decay of the nonlinear equation (6). From (56), we have to add a smallness condition on the initial data of system (6). This concludes the proof of Theorem 2 .

\section{SOME EXTENSIONS}

As mentioned in Remark 3, this method can be applied to stabilize other related KdV systems. In this Section, we focus in the linear control design because the nonlinear part of the argument is the same as in Section $\mathrm{V}$. More precisely, we show the equations that define the kernel functions corresponding to each case.

\section{A. Different boundary conditions}

In order to stabilize system

$$
\left\{\begin{array}{l}
u_{t}(t, x)+u_{x}(t, x)+u_{x x x}(t, x)=0 \\
u(t, 0)=\kappa(t), \quad u_{x}(t, L)=0, \quad u_{x x}(t, L)=0
\end{array}\right.
$$


we have to consider a kernel $k=k(x, y)$ satisfying on the triangle $\mathcal{T}:=\{(x, y) / x \in[0, L], y \in$ $[x, L]\}$ the equation

$$
\left\{\begin{aligned}
k_{x x x}(x, y)+k_{y y y}(x, y)+k_{x}(x, y)+k_{y}(x, y) & =-\lambda k(x, y), & & \text { in } \mathcal{T}, \\
k_{y y}(x, L) & =0, & & \text { in }[0, L], \\
k(x, x) & =0, & & \text { in }[0, L], \\
k_{x}(x, x) & =\frac{\lambda}{3}(L-x), & & \text { in }[0, L] .
\end{aligned}\right.
$$

The transformation

$$
w(x)=\Pi(u(x)):=u(x)-\int_{x}^{L} k(x, y) u(y) d y,
$$

map the solution $u=u(t, x)$ into the trajectory $w=w(t, x)$, solution of the target system

$$
\left\{\begin{array}{l}
w_{t}(t, x)+w_{x}(t, x)+w_{x x x}(t, x)+\lambda w(t, x)=0, \\
w(t, 0)=0, \quad w_{x}(t, L)=0, \quad w_{x x}(t, L)=0,
\end{array}\right.
$$

which is exponentially asymptotically stable for $\lambda>0$, with a decay at least equals to $\lambda$. By making the change of variable

$$
t=y-x, \quad s=x+y,
$$

and defining $G(s, t):=k(x, y)$, we get that $G$ is solution of

$$
\begin{aligned}
G(s, t) & =-\frac{\lambda}{6}(2 L-s)-2 \int_{0}^{t} G_{s}(s, \tau) d \tau-2 \int_{0}^{t} \int_{0}^{\tau} G_{s s}(s, \xi) d \xi d \tau \\
+ & \frac{1}{6} \int_{0}^{t} \int_{0}^{\tau} \int_{s}^{2 L-\xi}\left(-4 G_{s s s}(\eta, \xi)+2 G_{s}(\eta, \xi)+\lambda G(\eta, \xi)-12 G_{s s t}(\eta, \xi)\right) d \xi d \tau d \eta
\end{aligned}
$$

that can be studied by applying again the method of successive approximations.. 
B. Non-constant coefficient case

Given two functions $\gamma \in C^{1}([0, L])$ and $\delta \in C([0, L])$, we consider the non-constant coefficient linear $\mathrm{KdV}$ equation

$$
\left\{\begin{array}{l}
u_{t}(t, x)+\gamma(x) u_{x}(t, x)+u_{x x x}(t, x)+\delta(x) u=0 \\
u(t, 0)=\kappa(t), \quad u(t, L)=0, \quad u_{x}(t, L)=0
\end{array}\right.
$$

and the target system

$$
\left\{\begin{array}{l}
w_{t}(t, x)+\gamma(x) w_{x}(t, x)+w_{x x x}(t, x)+(\lambda+\delta(x)) w(t, x)=0 \\
w(t, 0)=0, \quad w(t, L)=0, \quad w_{x}(t, L)=0
\end{array}\right.
$$

which is exponentially asymptotically stable for $\lambda$ large enough. In fact,

$$
\lambda \geq \max _{x \in[0, L]}\left|\gamma^{\prime}(x)-2 \delta(x)\right|
$$

is a sufficient condition to ensure an exponential decay rate equals to $\lambda / 2$ for solutions of (64).

In this case, the kernel $k=k(x, y)$ to be considered in order to define the corresponding transformation $(59)$ is the solution of

$$
\left\{\begin{aligned}
k_{x x x}(x, y)+k_{y y y}(x, y)+\gamma(x) k_{x}(x, y)+\gamma(y) k_{y}(x, y) & =\alpha(x, y) k(x, y), & & \text { in } \mathcal{T}, \\
k(x, L) & =0, & & \text { in }[0, L], \\
k(x, x) & =0, & & \text { in }[0, L], \\
k_{x}(x, x) & =\frac{\lambda}{3}(L-x), & & \text { in }[0, L] .
\end{aligned}\right.
$$

where $\alpha$ is defined by

$$
\alpha(x, y)=-\left(\lambda+\gamma^{\prime}(y)+\delta(x)+\delta(y)\right)
$$


By using the change of variable (61), system (65) can be lead to the equation

$$
\begin{aligned}
G(s, t)=-\frac{\lambda t}{6}(2 L- & t-s) \\
& +\frac{1}{6} \int_{s}^{2 L-t} \int_{0}^{t} \int_{0}^{\tau}\left(2 G_{s s s}(\eta, \xi)+a_{1}(\eta, \xi) G_{s}(\eta, \xi)\right. \\
& \left.+a_{2}(\eta, \xi) G_{t}(\eta, \xi)+\alpha\left(\frac{\eta-\xi}{2}, \frac{\eta+\xi}{2}\right) G(\eta, \xi)\right) d \xi d \tau d \eta
\end{aligned}
$$

where

$$
a_{1}(\eta, \xi)=\gamma\left(\frac{\eta+\xi}{2}\right)+\gamma\left(\frac{\eta-\xi}{2}\right) \quad \text { and } \quad a_{2}(\eta, \xi)=\gamma\left(\frac{\eta+\xi}{2}\right)-\gamma\left(\frac{\eta-\xi}{2}\right)
$$

As before, this equation can be studied by applying the method of successive approximations.

\section{A non-zero equilibrium state}

We consider $u=u(t, x)$ solution of (6). If instead of linearizing this system around zero, we do that around a non-zero equilibrium state $z=z(x)$ solution of

$$
\left\{\begin{array}{l}
z^{\prime}(x)+z^{\prime \prime \prime}(x)+z(x) z^{\prime}(x)=0 \\
z(0)=U_{0}, \quad z(L)=0, \quad z^{\prime}(L)=0,
\end{array}\right.
$$

with $U_{0} \neq 0$, we have to consider the control system

$$
\left\{\begin{array}{l}
v_{t}(t, x)+(1+z(x)) v_{x}(t, x)+v_{x x x}(t, x)+z_{x}(x) v(t, x)=0, \\
v(t, 0)=h(t), \quad v(t, L)=0, \quad v_{x}(t, L)=0
\end{array}\right.
$$

where $v=v(t, x)$ and $h=h(t)$ are the first-order approximation of the state $(u(t, x)-z(x))$ and the control $\left(\kappa(t)-U_{0}\right)$, respectively. Thus, by using the control design in Subsection VI-B for non-constant coefficients, we can locally stabilizes system (6) around a non-zero equilibrium.

\section{CONCLuSions}

We have applied the backstepping method to build some boundary feedback laws, which locally stabilize the Korteweg-de Vries equation posed on a finite interval. Our control acts on the Dirichlet boundary condition at the left hand side of the interval where the system evolves. 
The closed-loop system is proven to be locally exponentially stable with a decay rate that can be chosen to be as large as we want. This approach allows to consider the KdV equation (6) with other boundary conditions and low order terms as $u_{x}$ or $u$ with non-constant coefficients depending on the space variable.

The situation where we act on the right end-point is different. If we consider homogeneous Dirichlet condition on the left and one or two control inputs at the right hand side of the interval, then we are not able to prove the backstepping method works with the transformation (12). Indeed, when imposing

$$
w_{t}+w_{x}+w_{x x x}+\lambda w=0
$$

on the target system, we get the following expression at $x=L$ to vanish

$$
k(x, L) u_{x x}(t, L)+k(x, L) u(t, L)+k_{y y}(x, L) u(t, L)-k_{y}(x, L) u_{x}(t, L) .
$$

As we do not have to our disposal $u_{x x}(t, L)$, the first term above arises the condition $k(x, L)=0$. Even if we do not care about the two last terms in (72), in order to keep $w(t, 0)=u(t, 0)=0$, we have to impose $k(0, y)=0$ for any $y \in(0, L)$. With these four boundary restrictions (the other two are on $k(x, x)$ ), the third order kernel equation satisfied by $k=k(x, y)$ becomes overdetermined. Therefore, it is not clear if such a function $k=k(x, y)$ exists.

A natural idea to deal with controls at $x=L$ is to use the transformation

$$
w(t, x)=u(t, x)-\int_{0}^{x} k(x, y) u(t, y) d y
$$

instead of (12). However, it is not clear if that approach works. In fact, if we do that, we have to deal now with the extra condition $k_{y}(x, 0)=0$ for any $x \in(0, L)$. This is due to the fact that when imposing $w_{t}+w_{x}+w_{x x x}+\lambda w=0$ on the target system, we get the extra term $u_{x}(t, 0) k_{y}(x, 0)$ to be cancelled. As previously, this fourth restriction gives an overdetermined kernel equation for $k=k(x, y)$. Moreover, the existence of critical lengths when only one control is considered at the right end-point suggests that either the existence of the kernel or the 
invertibility of the corresponding map $\Pi$ should fail for some spatial domains.

Two related problems that remain still open are the boundary global or semi-global stabilization and the output feedback control problem. The boundary global stabilization is hard because it is needed a really nonlinear design as in [14] for the $\mathrm{KdV}$ equation with periodic boundary conditions. Concerning the output feedback control problem, we believe it could be solved by applying the backstepping approach in order to built some observers as done in [28], [12] for the heat and the wave equations respectively.

Acknowledgements. This work has been done while the first author was visiting the Laboratoire Jacques-Louis Lions at Université Pierre et Marie Curie in Paris. He thanks this institution for its hospitality.

\section{REFERENCES}

[1] E. CERPA, Exact controllability of a nonlinear Korteweg-de Vries equation on a critical spatial domain, SIAM J. Control Optim., 46 (2007), 877-899.

[2] E. CERPA, E. CRÉPEAU, Boundary controllability for the nonlinear Korteweg-de Vries equation on any critical domain, Ann. Inst. H. Poincaré Anal. Non Linéaire, 26 (2009), 457-475.

[3] E. Cerpa, E. Crépeau, Rapid exponential stabilization for a linear Korteweg-de Vries equation, Discrete Contin. Dyn. Syst. Ser. B 11 (2009), no. 3, 655-668.

[4] J.-M. Coron, Control and nonlinearity, Mathematical Surveys and Monographs, 136, American Mathematical Society, Providence, RI, 2007. xiv+426 pp.

[5] J.-M. Coron, E. CréPeAu, Exact boundary controllability of a nonlinear KdV equation with critical lengths, J. Eur. Math. Soc. (JEMS), 6 (2004), 367-398.

[6] J.-M. Coron, B. D’AndrÉA-Novel, Stabilization of a rotating body beam without damping, IEEE Trans. Automat. Control 43 (1998), no. 5, 608-618.

[7] O. Glass, S. Guerrero, Some exact controllability results for the linear KdV equation and uniform controllability in the zero-dispersion limit, Asymptot. Anal. 60 (2008), 61-100.

[8] O. Glass, S. Guerrero, Controllability of the Korteweg-de Vries equation from the right Dirichlet boundary condition, Systems Control Lett. 59 (2010), 390-395.

[9] D. L. Kleinman, An easy way to stabilize a linear control system, IEEE Trans. Automat. Control, 15 (1970), 692.

[10] V. KomORNIK, Rapid boundary stabilization of linear distributed systems, SIAM J. Control Optim., 35 (1997), $1591-1613$.

[11] M. Krstic, I. Kanellakopoulos, P. Kokotovic, "Nonlinear and Adaptive Control Design", John Wiley and Sons, 1995. 
[12] M. Krstic, B. Z. Guo, A. Balogh, A. Smyshlyaev, Output-feedback stabilization of an unstable wave equation, Automatica, 44 (2008), 63-74.

[13] M. Krstic, A. Smyshlyaev, Boundary control of PDEs. A course on backstepping designs. Advances in Design and Control, 16. Society for Industrial and Applied Mathematics (SIAM), Philadelphia, PA, 2008.

[14] C. Laurent, L. Rosier, B.-Y. Zhang, Control and stabilization of the Korteweg-de Vries equation on a periodic domain, Comm. Partial Differential Equations 35 (2010), 707-744.

[15] F. Linares, A.F. PAzoto, On the exponential decay of the critical generalized Korteweg-de Vries with localized damping, Proc. Amer. Math. Soc. 135 (2007), 1515-1522.

[16] W. LIU, Boundary feedback stabilization of an unstable heat equation, SIAM J. Control Optim., 42 (2003), $1033-1043$.

[17] W. LiU, M. KRSTIC, Backstepping boundary control of Burgers' equation with actuator dynamics, Systems Control Lett. 41 (2000), no. 4, 291-303.

[18] D. L. LuKES, Stabilizability and optimal control, Funkcial. Ekvac., 11 (1968), 39-50.

[19] A. F. PAzoto, Unique continuation and decay for the Korteweg-de Vries equation with localized damping, ESAIM Control Optim. Calc. Var. 11 (2005), 473-486.

[20] G. Perla Menzala, C. F. Vasconcellos, And E. Zuazua, Stabilization of the Korteweg-de Vries equation with localized damping, Quarterly of Applied Mathematics Vol. LX (2002), 111-129.

[21] L. RosIER, Exact boundary controllability for the Korteweg-de Vries equation on a bounded domain, ESAIM Control Optim. Calc. Var., 2 (1997), 33-55.

[22] L. RosIER, Control of the surface of a fluid by a wavemaker, ESAIM Control Optim. Calc. Var. 10 (2004), 346-380.

[23] L. Rosier, B.-Y Zhang, Global Stabilization of the generalized Korteweg-de Vries Equation Posed on a finite Domain, SIAM J. Control Optim. 45 (2006), 927-956.

[24] D. L. Russell, B.-Y. Zhang,Exact controllability and stabilizability of the Korteweg-de Vries equation, Trans. Amer. Math. Soc. 348 (1996), 3643-3672.

[25] M. Slemrod, A note on complete controllability and stabilizability for linear control systems in Hilbert space, SIAM J. Control, 12 (1974), 500-508.

[26] A. Smyshlyaev, E. Cerpa, M. Krstic, Boundary stabilization of a 1-D wave equation with in-domain antidamping, SIAM J. Control Optim. 48 (2010), no. 6, 4014-4031.

[27] A. Smyshlyaev, M. KRstic, Closed-form boundary state feedbacks for a class of 1-D partial integro-differential equations, IEEE Trans. Automat. Control, 49 (2004), 2185-2202.

[28] A. Smyshlyaev, M. Krstic, Backstepping observers for a class of parabolic PDEs, Systems Control Lett. 54 (2005), no. 7, 613-625.

[29] A. Smyshlyaev, M. KRStic, Boundary control of an anti-stable wave equation with anti-damping on the uncontrolled boundary, Systems Control Lett. 58 (2009), no. 8, 617-623.

[30] J. M. URQUIZA, Rapid exponential feedback stabilization with unbounded control operators, SIAM J. Control Optim., 43 (2005), 2233-2244. 